Review Article

\title{
Is the Origin and Emergence of SARS-CoV-2 Ingenuous?
}

\author{
Sajal Bhattacharya', Rina Tilak $^{2}$, Chandrima Bose ${ }^{3}$, Shakya Sinha ${ }^{4}$
}

${ }^{1}$ Professor (Associate), ${ }^{3,4}$ Research Student, Department of Zoology, Asutosh College (University of Calcutta), Kolkata, West Bengal, India.

${ }^{2}$ Scientist ' $G$ ', Department of Community Medicine, Armed Forces Medical College, Pune, Maharashtra, India.

DOI: https://doi.org/10.24321/0019.5138.202162

\section{I $\quad \mathbf{N} \quad \mathbf{F} \quad \mathbf{O}$}

\section{Corresponding Author:}

Rina Tilak, Department of Community Medicine, Armed Forces Medical College, Pune, Maharashtra, India.

E-mail Id:

rinatilak@hotmail.com

\section{Orcid Id:}

https://orcid.org/0000-0003-3781-0210

How to cite this article:

Bhattacharya S, Tilak R, Bose C, Sinha S. Is the Origin and Emergence of SARS-CoV-2 Ingenuous?

J Commun Dis. 2021;53(3):232-235.

Date of Submission: 2021-09-03

Date of Acceptance: 2021-09-29

\section{$\begin{array}{llllllll}\mathbf{A} & \mathbf{B} & \mathbf{S} & \mathbf{T} & \mathbf{R} & \mathbf{A} & \mathbf{C} & \mathbf{T}\end{array}$}

The inability to identify the source of origin of SARS-CoV-2 even after more than twenty months of its emergence is intriguing and challenges the scientists and the public health personnel alike. Apprehension has been raised in certain quarters that some sort of human interference has taken place in the already dynamic gene pool of coronaviruses, which is a matter of concern. The need to have a scientific audit is paramount to unearth the real narrative about its origin as the precipitous assumption based on reports of human case incidences of infectious respiratory viral diseases having pandemic potential from a specific region is scientifically premature and it does not conclusively confirm the region to be the place of origin of the viral pathogen. Delineation of the source of origin of SARS-CoV-2 is vital for formulating strategies for the prevention of future outbreaks of viral zoonotic diseases if any, development of effective candidate vaccines, and designing target specific drugs. Further, this will put to rest the controversy about the origin and emergence of SARS-CoV-2 and create an ambience for cooperative functioning among the global stakeholders. It is pertinent to have comprehensive scrutiny of the laboratories conducting experimental research on coronaviruses particularly bat and other suspected mammalian betacoronaviruses to avert such calamitous situations in future. Mitigation of the disastrous effects of the COVID-19 pandemic is a global responsibility and necessitates joint efforts from all stakeholders across the globe.

Keywords: SARS-CoV-2, Zoonotic Diseases, COVID-19 Pandemic, Bats

\section{Introduction}

The emergence of SARS-CoV-2 has caused the outbreak of the COVID-19 pandemic and created a stir among the scientific fraternity with millions of deaths across the globe. Viruses and their reservoir hosts have co-existed through co-evolution since time immemorial and maintained an ecological equilibrium. But in recent times, the world is witnessing the emergence and re-emergence of several viral diseases with epidemic and pandemic potentials. This could largely be a result of rampant deforestation, landuse changes, rapid urbanization, expansion of agricultural land and climate change subsequently disturbing and 
altering the ecological niche of reservoir hosts and making them acutely vulnerable. As a consequence, the ecological barrier between man and the reservoirs of several known or unknown diseases are getting narrower, thereby changing the transmission dynamics of these diseases. ${ }^{1,2}$ The emergence of the novel coronavirus (SARS-CoV-2) might not be an exception to this as postulated by several groups of scientists. ${ }^{2,3}$ Further, in today's highly globalised and wellconnected world, the transfer and transport of pathogens and reservoir hosts is not unusual. Consequently, emerging infectious diseases, such as COVID-19, are spreading like wildfire. The initial cases of the novel coronavirus were reported from the Hubei province of Wuhan city in China. ${ }^{4}$ ${ }^{5}$ Bats are assumed to be the most likely reservoir hosts of SARS-CoV-2 based on the sequence similarity between the bat CoV RaTG13 and SARS-CoV-2. ${ }^{3,6}$ However, even after more than twenty months of the emergence of the SARSCoV-2, there has been no convincing evidence to establish bat and other intermediate hosts as the direct source for its human zoonotic transmission. ${ }^{7}$ In this murky circumstance, there are diverse and conflicting views about the origin of SARS-CoV-2. WHO Director-General in his opening remarks at the 'Member State Information Session on Origins' about SARS-CoV-2 said, "finding the origins of this virus is a scientific exercise that must be kept free from politics." ${ }^{8}$ Our focus of analysis in this article is to seek the truth regarding the origin of SARS-CoV-2 without any bias by analyzing and interpreting the available scientific literature.

\section{Nebulous Anatomy and Infectious Behavior of SARS-CoV-2}

Detailed research on SARS-CoV-2 has revealed the role of S1 subunit of the spike glycoprotein (S) in binding the ACE-2 receptors via its receptor-binding domain (RBD) for the infection. ${ }^{9}$ Interestingly, despite having a $96.2 \%$ sequence homology to bat CoV RaTG13, ${ }^{10}$ SARS-CoV-2 has shown an enhanced ability to bind to the human ACE2 (Angiotensin-converting enzyme 2), while bats tested for the virus exhibited poor infectivity. ${ }^{11}$ The plausible reason for such a paradoxical phenomenon still remains unanswered; however, a likely explanation for the same could be attributed to the high mutability of RNA viruses and a high rate of adaptation of this virus. The reason for the poor infectivity of SARS-CoV-2 in bats is yet to be understood and demands a comprehensive investigation.

The acquisition of a 'Furin cleavage site' of SARS-CoV-2 is of pivotal importance as several studies have confirmed that this polybasic Furin cleavage site has rendered human infection competence to SARS-CoV-2. ${ }^{12,13}$ The nucleotide sequence insert of TCCTCGGCGGGC codes for the PRRA amino acid sequence, the building block for this cleavage site. It is intriguing that the coronavirus strains viz. bat RaTG13 and Pangolin MP789 that share the most similarities with the SARS-CoV-2 RBD do not have these twelve nucleotides in the genomic open reading frame. ${ }^{12}$ This eliminates any chances of insertion of this sequence by polymerase slippage. Researchers are also sceptical about the insertion being a result of recombination events. ${ }^{14}$ Nevertheless, the presence of Furin cleavage sites is not uncommon in coronaviruses and has been documented for other human coronaviruses such as MERS-CoV, HCoVHKU1 and HCoV-OC43. ${ }^{15}$ Further research in this direction is needed to find any consolidating explanation for the occurrence of such Furin cleavage sites in SARS-CoV-2 spike protein. Apart from this, another facet of SARS-CoV-2 is the emergence of an unusually high frequency of variants. ${ }^{16}$ This brings us back to question the ingenuity of the emergence of SARS-CoV-2.

\section{Tracing the Origin}

Virtually all the natural human coronaviruses are of zoonotic origin ${ }^{1}$ and almost all of them are postulated to be derived from the bat reservoirs where they got hosted and evolutionarily shaped. ${ }^{10}$ SARS-CoV-2 has also been thought to have originated from bats owing to its high sequence similarity with bat CoV RaTG13. ${ }^{10}$ Some researchers have indicated the Huanan seafood market in Wuhan to be a possible site of the first zoonotic transfer of the virus from wild animals to humans. ${ }^{2}$ However, a school of researchers consider that the virus might have been genetically manipulated or tampered with, thereby 'humanizing' what we primarily thought was a bat virus. ${ }^{7}$ 12 Possibilities of CRISPR based interference of an already dynamic gene pool of coronaviruses is a concerning avenue for evaluation and scientific assessment. ${ }^{17}$

The WHO publication on SARS indicated that in 2003 the SARS-CoV emerged from China and went on its way to become an epidemic, eventually spreading to four countries. ${ }^{18}$ Among several apprehensions about the origin of SARS-CoV-2, its emergence from a Chinese laboratory has been widely speculated and discussed. ${ }^{7}$ The Wuhan Institute of Virology (WIV), which conducts research on coronaviruses, is also situated in Wuhan city, where the first human outbreak of SARS-CoV-2 was reported. The likely possibility of an accidental laboratory release or leakage of the virus regardless of all bio-safety measures has been a hotly debated topic. Such incidents are not rare events and have been documented before. ${ }^{12}$ According to the WHO Director-General, “... audits of relevant laboratories and research institutions operating in the area of the initial human cases identified in December 2019" should be done. ${ }^{8}$ In all fairness, we must admit that coronavirus related research is not exclusively carried out in Chinese laboratories. Researches on coronaviruses are carried out in several countries globally. ${ }^{19,20}$ In fact bats, the most notable reservoir hosts for several coronaviruses and the 
postulated source of SARS-CoV- $2^{1,6}$, are also found in all the habitable continents of the world encompassing many countries including China. Therefore, a comprehensive scrutiny of laboratories conducting experimental research on coronaviruses especially bat and other suspected mammalian beta-coronaviruses is needed. Furthermore, the reports of human case incidences of infectious respiratory viral diseases having pandemic potential from a specific region do not conclusively confirm the region to be the place of origin of the viral pathogen. Therefore, it is debatable that SARS-CoV-2 originated or emerged from China solely based on first information reports of human cases from there, especially keeping in mind the fact that the world today is a well-connected 21st century global village. It is, nevertheless, crucial to precisely identify the site of origin of SARS-CoV-2 and its subsequent emergence and global spread timeline for better understanding and futuristic planning for the prevention of pandemics. The findings of the stated scientific audit should certainly be confirmed, reconfirmed, accepted and approved by the globally recognized and authorized scientific bodies. At this juncture, it is, however, difficult to arrive at a definitive logical conclusion regarding the source of origin of this SARSCoV-2. It would be unwise to indulge in any speculations or assumptions without any concrete and convincing scientific evidence-based research.

\section{Conclusion}

Owing to diverse and conflicting views about the origin and emergence of SARS-CoV-2, the authors at this juncture are reluctant to draw any conclusion regarding the issue. A comprehensive scientific evidence-based investigation is an imperative necessity to unravel the real narrative which in turn would prove vital for formulating strategies for the prevention of future outbreaks of viral zoonotic diseases if any, development of effective candidate vaccines and designing target specific drugs. Further, this will put to rest the controversy regarding the origin and emergence of SARS-CoV-2 and create an ambience for cooperative functioning among the stakeholders across the globe.

\section{Conflict of Interest: None}

\section{References}

1. Bhattacharya S, Sinha S, Tilak R, Mardihusodo SJ. The relationship between bats and human coronavirus: An exploratory review. J Health Soc Sci. 2020;5(2):219-30. [Google Scholar]

2. Huang $X$, Zhang C, Pearce R, Omenn GS, Zhang Y. Identifying the zoonotic origin of SARS-CoV-2 by modeling the binding affinity between the spike receptor-binding domain and host ACE2. J Proteome Res. 2020 Dec;19(12):4844-56. [PubMed] [Google Scholar]
3. Lau SKP, Luk HKH, Wong ACP, Li KSM, Zhu L, He Z, Fung J, Chan TTY, Fung KSC, Woo PCY. Possible bat origin of severe acute respiratory syndrome coronavirus 2 . Emerg Infect Dis. 2020 Jul;26(7):1542-7. [PubMed] [Google Scholar]

4. Malik YA. Properties of coronavirus and SARS-CoV-2. Malays J Pathol. 2020 Apr;42(1):3-11. [PubMed] [Google Scholar]

5. Huang C, Wang Y, Li X, Ren L, Zhao J, Hu Y, Zhang L, Fan G, Xu J, Gu X, Cheng Z, Yu T, Xia J, Wei Y, Wu W, Xie X, Yin W, Li H, Liu M, Xiao Y, Gao H, Guo L, Xie J, Wang G, Jiang R, Gao Z, Jin Q, Wang J, Cao B. Clinical features of patients infected with 2019 novel coronavirus in Wuhan, China. Lancet. 2020 Feb;395(10223):497-506. [PubMed] [Google Scholar]

6. Zhou H, Ji J, Chen X, Bi Y, Li J, Wang Q, Hu T, Song H, Zhao R, Chen Y, Cui M, Zhang Y, Hughes AC, Holmes $\mathrm{EC}$, Shi W. Identification of novel bat coronaviruses sheds light on the evolutionary origins of SARS-CoV-2 and related viruses. Cell. 2021 Aug;184(17):4380-91. e14. [PubMed] [Google Scholar]

7. Balaram P. The murky origins of the coronavirus SARSCoV-2, the causative agent of the COVID-19 pandemic. Curr Sci. 2021;120(11):1663-6. [Google Scholar]

8. World Health Organization [Internet]. WHO DirectorGeneral's opening remarks at the member state information session on origins; 2021 [cited 2021 Jul 17]. Available from: https://www.who.int/directorgeneral/speeches/detail/who-director-general-sopening-remarks-at-the-member-state-informationsession-on-origins.

9. Elrashdy F, Redwan EM, Uversky VN. Why COVID-19 transmission is more efficient and aggressive than viral transmission in previous coronavirus epidemics? Biomolecules. 2020 Sep;10(9):1312. [PubMed] [Google Scholar]

10. Tang X, Wu C, Li W, Song Y, Yao X, Wu X, Duan Y, Zhang H, Wang Y, Qian Z, Cui J, Lu J. On the origin and continuing evolution of SARS-CoV-2. Natl Sci Rev. 2020;7(6):10123. [Google Scholar]

11. Liu K, Tan S, Niu S, Wang J, Wu L, Sun H, Zhang Y, Pan X, Qu X, Du P, Meng Y, Jia Y, Chen Q, Deng C, Yan J, Wang HW, Wang Q, Qi J, Gao GF. Cross-species recognition of SARS-CoV-2 to bat ACE2. Proc Natl Acad Sci USA. 2021 Jan;118(1):e2020216118. [PubMed] [Google Scholar]

12. Segreto $R$, Deigin $Y$. The genetic structure of SARS-CoV-2 does not rule out a laboratory origin: SARS-COV-2 chimeric structure and furin cleavage site might be the result of genetic manipulation. Bioessays. 2021 Mar;43(3):e2000240. [PubMed] [Google Scholar]

13. Hoffmann M, Kleine-Weber HA, Pöhlmann S. A multibasic cleavage site in the spike protein of SARSCoV-2 is essential for infection of human lung cells. 
Mol Cell. 2020 May;78:779-84.e5. [PubMed] [Google Scholar]

14. Seyran M, Pizzol D, Adadi P, El-Aziz TMA, Hassan SS, Soares A, Kandimalla R, Lundstrom K, Tambuwala M, Aljabali AAA, Lal A, Azad GK, Choudhury PP, Uversky VN, Sherchan SP, Uhal BD, Rezaei N, Brufsky AM. Questions concerning the proximal origin of SARS-CoV-2. J Med Virol. 2021;93(3):1204-6. [PubMed] [Google Scholar]

15. Wu Y, Zhao S. Furin cleavage sites naturally occur in coronaviruses. Stem Cell Res. 2020 Dec;50:102115. [PubMed] [Google Scholar]

16. Wang R, Chen J, Gao K, Hozumi Y, Yin C, Wei GW. Analysis of SARS-CoV-2 mutations in the United States suggests presence of four substrains and novel variants. Commun Biol. $2021 \mathrm{Feb} ; 4(1): 228$. [PubMed] [Google Scholar]

17. Chatterjee R, Bhattacharya S. Could novel corona virus (SARS-CoV-2) be the evolving face of a new generation of genetically complex epidemiological challenge? Malays J Med Res. 2020;4(2):49-52. [Google Scholar]

18. World Health Organization [Internet]. Severe Acute Respiratory Syndrome (SARS); 2021 [cited 2021 Jul 4]. Available from: https://www.who.int/health-topics/ severe-acute-respiratory-syndrome\#tab=tab_1.

19. Goffard A, Demanche C, Arthur L, Pinçon C, Michaux J, Dubuisson J. Alphacoronaviruses detected in French bats are phylogeographically linked to coronaviruses of European bats. Viruses. 2015 Dec;7:6279-90. [PubMed] [Google Scholar]

20. Smith CS, de Jong CE, Meers J, Henning J, Wang L, Field HE. Coronavirus infection and diversity in bats in the Australasian region. Ecohealth. 2016 Mar;13(1):72-82. [PubMed] [Google Scholar] 